ARTICULO DE INVESTIGACION

\title{
Evaluación de programas de secado para madera de chalamite (Pinus pseudostrobus)
}

\author{
Evaluation of drying schedules for chalamite wood \\ (Pinus pseudostrobus)
}

\author{
Laura V. Aquino-González 1, Juan Rodríguez-Ramírez1, \\ Lilia L. Méndez-Lagunas ${ }^{1}$ y Sadoth Sandoval-Torres ${ }^{1}$
}

\section{RESUMEN}

\begin{abstract}
El objetivo de este estudio es analizar el secado técnico convencional de madera de chalamite (Pinus pseudostrobus) utilizando un diseño factorial 22. Se aplicaron dos programas de secado: uno acelerado con temperatura inicial de $76{ }^{\circ} \mathrm{C}$ y final de $87^{\circ} \mathrm{C}$ y otro suave con temperatura inicial de $60{ }^{\circ} \mathrm{C}$ y final de $82{ }^{\circ} \mathrm{C}$, combinado con dos velocidades de aire: $2,05 \mathrm{~ms}^{-1}$ y $3,35 \mathrm{~ms}^{-1}$. Las variables respuesta evaluadas fueron: tiempo de secado y calidad de la madera seca. De acuerdo con los resultados el tiempo de secado es afectado por la intensidad del programa, y la calidad de la madera depende del programa de secado y de la velocidad del aire. Estadísticamente se observó que un programa de secado con temperaturas de $76{ }^{\circ} \mathrm{C}$ a $87^{\circ} \mathrm{C}$ y velocidad de aire de $3.35 \mathrm{~ms}^{-1}$ redujo el tiempo de secado y la generación de defectos en la madera seca.
\end{abstract}

PALABRAS CLAVE:

Defectos, diseño factorial, tiempo de secado, velocidad del aire.

\begin{abstract}
The aim of this study is to analyze the kiln-drying of chalamite wood (Pinus pseudostrobus) using a $2^{2}$ factorial design. Two drying schedule were applied: the first one was an intense schedule with temperatures from $76^{\circ} \mathrm{C}$ to $87^{\circ} \mathrm{C}$, and the second one a soft schedule with temperatures from $60{ }^{\circ} \mathrm{C}$ to $82{ }^{\circ} \mathrm{C}$; these schedules were applied with two air velocities: $2,05 \mathrm{~ms}^{-1}$ and 3,35 ms-1. Drying time and quality of wood were the responses evaluated. According to the results, the drying time is affected by the schedule intensity, and the wood quality depends on the drying schedule and air velocity. Statistically, it was observed that a drying schedule with temperatures from $76{ }^{\circ} \mathrm{C}$ to $87^{\circ} \mathrm{C}$ and air velocity of $3,35 \mathrm{~ms}^{-1}$ reduces the drying time and defects development in wood.
\end{abstract}

KEYWORDS:

Defects, factorial design, drying time, air velocity.

Centro Interdisciplinario de Investigación para el Desarrollo Integral Regional, IPN Unidad Oaxaca. Laboratorio de Tecnologías Agroalimentarias. Hornos 1003, Santa Cruz Xoxocotlán, CP 71230, Oaxaca, Oaxaca, México.

e-mail: lauvicka@hotmail.com, jrodrigr@hotmail.com, mendezll@hotmail.com, sadothsan@yahoo.com 


\section{INTRODUCCIÓN}

Los madereros y fabricantes de productos maderables en México mencionan que las mejores maderas de pino son las de Durango, Chihuahua y Oaxaca debido a sus características físicas como: color, dureza, porosidad, durabilidad, y homogeneidad, (USAID, 2007). Sin embargo; los madereros del centro, norte y sur del país consideran que la madera Oaxaqueña es de las más caras del mundo debido a que el transporte en el estado es de los más caros, lo cual incrementa el costo de la madera. Otro factor que incrementa el costo de la madera es la calidad, la cual se determina en función de la superficie utilizable ó libre de defectos (nudos, bolsas de resina, fisuras, rajaduras, manchas y otros).

Una opción para reducir los costos e incrementar la calidad de la madera es la comercialización de madera con un contenido de humedad $(\mathrm{CH})$ del $10 \%$, obtenida con el secado solar ó el secado técnico convencional (Wiberg et al, 2000); el contenido de humedad se define como el peso de agua presente en una pieza de madera (en condición verde), expresado en función del peso anhidro (en condición seca). Sin embargo por las limitantes económicas de Oaxaca, el secado de madera se realiza en su mayoría al aire libre y sin control, debido a que este proceso tiene una duración de 5 a 7 meses hasta alcanzar un $\mathrm{CH}$ del 12 al $10 \%$ se generan contracciones y deformaciones que reducen la calidad y valor agregado de estas maderas. El secado artificial ó estufado constituye una fase del proceso de industrialización de la madera aserrada, en este proceso se combinan temperaturas de bulbo seco (TBS) y bulbo húmedo (TBH), humedades relativa (HR \%) y velocidades de aire (Simpson, 1994; Carlsson, 2002; Solís et al, 2003; Aytekin et al., 2009).
Un programa de secado se establece de acuerdo con la especie, espesor y uso final de la madera; en este proceso se considera la relación entre el balance de calor transferido por el flujo de aire a la superficie del material y del transporte de agua del centro a la superficie del mismo. El proceso de secado consta de tres fases determinadas por la variación de la velocidad del secado: una fase de calentamiento una fase de secado y una de acondicionamiento; el movimiento del agua a través de la madera dependerá de las propiedades físicas del material y la velocidad del secado, la cual es controlada por las condiciones externas (temperaturas, velocidad de aire, humedad relativa); en la fase higroscópica, la velocidad de secado es controlada por la resistencia interna; en la tercera fase el contenido de humedad de la madera se uniformiza hasta alcanzar el equilibrio (Kollman y Cotê, 1968; Rosen, 1983; Jankowsky, 1995).

La madera seca sufre cambios en sus propiedades físicas que producen esfuerzos, contracciones y grietas (Sandoval-Torres, 2009). La contracción causa defectos tales como: endurecimiento, colapso celular, grietas, rajaduras y torceduras (Álvarez y Fernández, 1992; Langrish et al., 1997; Blakemore, 2008; Bustos et al. 2009), estos pueden ser ponderados para determinar la calidad del secado (Kauman y Mittak, 1966). De acuerdo a Brooke y Langrish (1997), el estrés formado dentro de la madera produce una contracción y expansión térmica provocada por los gradientes de humedad y temperatura.

\section{OBJETIVO}

Evaluar programas de secado técnico convencional para madera de pino chalamite (Pinus pseudostrobus) y analizar su efecto en el tiempo de secado y en la calidad de la madera. 


\section{METODOLOGÍA}

\section{Selección y preparación de la muestra}

En este estudio se utilizó madera de pino chalamite (Pinus pseudostrobus) muestreada en el Distrito de San Miguel Aloapan ubicado de la Sierra Norte de Oaxaca. Solo se seleccionó un árbol de esta especie debido a que las pruebas de de secado se realizaron en un secador prototipo y el objetivo principal fue obtener resultados preliminares del secado de esta especie. El árbol seleccionado tenía una altura de 20 metros, con diámetro de $60 \mathrm{~cm}$ con corteza, y $54 \mathrm{~cm}$ sin corteza. El tronco se seccionó en 5 trozas, de las cuales se obtuvieron 12 tablas con dimensiones de $2,5 \mathrm{~cm} \times 20 \mathrm{~cm}$ x $256 \mathrm{~cm}$ (Figura 1). Por cada tabla se obtuvieron 4 especímenes de 2,5 cm x $20 \mathrm{~cm}$ x $50 \mathrm{~cm}$ y 2 probetas de $2,5 \mathrm{~cm}$ x 2,5 $\mathrm{cm} \times 20 \mathrm{~cm}$. para determinar el contenido de humedad. En total se obtuvieron 32 especímenes y 64 probetas.

Los especímenes y probetas se pesaron inmediatamente después del corte en una balanza Navigator marca Ohaus de $8000 \mathrm{~g} \pm 0,5 \mathrm{~g}$. El peso medido se consideró como peso en verde. Los especímenes se sellaron por los costados con silicón y se mantuvieron en una solución de pentaclorofenato de sodio, para evitar la pérdida de humedad y evitar la aparición de mancha azul en dichos especímenes.

\section{Determinación de Contenido de Humedad $(\% \mathrm{CH})$}

Las probetas obtenidas de cada muestra, se secaron en una estufa de vacío Marca Shelab a una temperatura de $103 \pm 2{ }^{\circ} \mathrm{C}$ hasta alcanzar un peso constante.

El contenido de humedad $(\mathrm{CH})$ se calculó con la metodología propuesta por Simpson (1991), usando la siguiente relación:

$$
C H=\left\lfloor\frac{P_{h}-P_{s}}{P_{s}} \times 100\right\rfloor \%
$$

Durante el proceso de secado el peso seco calculado de las especímenes se determinó con la siguiente relación:

$$
P S C=\frac{P_{h}}{100+C H} \times 100
$$

\section{Diseño de experimentos y determina- ción de variables respuesta}

Para establecer los programas de secado se siguió la metodología propuesta por Simpson (1996). Para elaborar el programa de secado se midió la densidad de la madera en un esteropicnómetro marca Quantachrome modelo SPY-5DC, la densidad real fue de $1,4603 \mathrm{~g} / \mathrm{cm}^{3}$; con este valor se seleccionó la temperatura

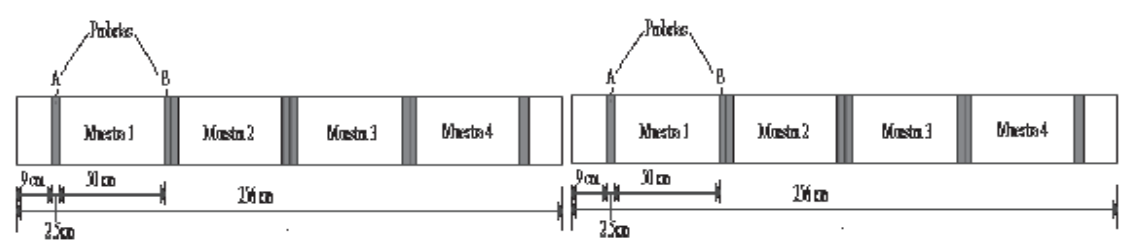

Figura 1. Corte de la tabla para obtener 4 especímenes para secar y 8 probetas para $\mathrm{CH}$. 
de bulbo seco (TBS), temperatura de bulbo húmedo $(T B H)$. Con el Contenido de Humedad Inicial $\left(\mathrm{CH}_{i}\right)$ se seleccionaron las condiciones del programa de secado: Humedad relativa $(\% H R)$, contenido de humedad en equilibrio ( $C H E$ ), y coeficiente de secado (CS). El coeficiente de secado se calcula dividiendo el contenido de humedad en equilibrio entre el contenido de humedad de la muestra en la etapa correspondiente. En las tablas 1 y 2 se muestran los programas de secado aplicados en este estudio.

Los especímenes de madera con dimensiones $2,5 \mathrm{~cm} \times 20 \mathrm{~cm} \times 50 \mathrm{~cm}$ se colocaron dentro de un secador tipo túnel (Rodríguez et al., 2001), en donde el aire fluye uniforme y longitudinalmente entre las tablas. Este flujo de aire se genera con un ventilador centrífugo marca ARME y se controla con un variador de frecuencia marca ABB. Las velocidades de utilizadas fueron $2,05 \mathrm{~ms}^{-1}$ y $3,35 \pm 0,05 \mathrm{~ms}^{-1}$.

El sistema se humidificó con un generador de vapor marca Anghinetti modelo TV/62F; la humedad relativa ( $\% H R$ ) y temperatura se midieron con un sensor humicap marca Vaisala modelo HMP234 colocado dentro del túnel, el calentamiento se controló con un controlador PID regulando la temperatura de acuerdo a las etapas del programa de secado (Tabla 1 y 2).

Las condiciones del secado se aplicaron siguiendo un diseño de experimentos factorial 22 , en cual se combinan dos programas de secado (acelerado y suave) y dos velocidades de aire $(2,05$ $\mathrm{ms}^{-1}$ y $3,35 \mathrm{~ms}^{-1}$ ), obteniendo un total de 8 pruebas experimentales (Tabla 3 ). Para cada prueba del diseño se usaron 4 especímenes de $2,5 \times 20 \times 50 \mathrm{~cm}$. El secado termina cuando el contenido de humedad promedio es del $10 \%$ en los especímenes.
En México existen normas para la clasificación de la madera aserrada la cual se realiza de acuerdo a la presencia de nudos, fibra desviada, defectos de crecimiento, madera juvenil entre otros, sin embargo aun no se ha establecido un método estandarizado por lo cual cada región establece sus propios procedimientos. Por tal motivo, en el presente estudio se utilizó la Norma Chilena 993 EOf 72 (1993), que en la actualidad ha servido para cuantificar la calidad de la maderas suaves y latifoliadas en diversos estudios (Guerrero, 1980; Fuentes, 1991; Sandoval et al, 2004; Pérez et al, 2007) y que a la vez es una metodología de fácil comprensión por los operarios secadores en el estado de Oaxaca.

De acuerdo con la Norma Chilena, para cuantificar la calidad de la madera seca se manejaron 4 valores porcentuales máximos permisibles ó grados de intensidad de defectos correspondientes a la dimensión de la tabla estufada; los defectos se determinaron con la metodología de Kauman y Mittak (1966): se contabilizaron las rajaduras y grietas con mayor longitud y se realizó la sumatoria por muestra; para medir arqueadura, torcedura, acanaladura y encorvadura la muestra se colocó en superficie lisa y se registró la distancia que se forma entre el defecto y la superficie. El valor obtenido por defecto se multiplicó por el factor de desclasificación (Tabla 4) para determinar la media ponderada de desclasificación o grado total de desclasificación (MPD), en el Tabla 5 se ubicó la media para establecer el índice de calidad de las tablas secas (ICS) y las condiciones de operación del secado (CDS). El tiempo de secado y calidad de la madera seca se evaluaron con un análisis de varianza y un alfa de 0,05 usando NCSS 2001. 
Tabla 1. Programa de secado acelerado.

\begin{tabular}{ccccccc}
\hline Etapa & $\% \mathrm{CH}$ & $\mathrm{TBS}\left({ }^{\circ} \mathrm{C}\right)$ & $\mathrm{TBH}\left({ }^{\circ} \mathrm{C}\right)$ & $\% \mathrm{HR}$ & $\mathrm{CHE}$ & $\mathrm{CS}$ \\
\hline 0 & - & 76,6 & 76,6 & 100 & - & - \\
1 & $120-70$ & 76,6 & 65,5 & 60 & 7,8 & 0,111 \\
2 & $70-60$ & 76,6 & 62,7 & 52 & 6,7 & 0,111 \\
3 & $60-50$ & 76,6 & 60 & 45 & 5,7 & 0,114 \\
4 & $50-40$ & 76,6 & 57,2 & 39 & 5,1 & 0,127 \\
5 & $40-35$ & 76,6 & 57,2 & 39 & 5,1 & 0,145 \\
6 & $35-30$ & 76,6 & 57,2 & 39 & 5,1 & 0,170 \\
7 & $30-25$ & 82,2 & 62,7 & 41 & 5,0 & 0,200 \\
8 & $25-20$ & 82,2 & 62,7 & 41 & 5,0 & 0,200 \\
9 & $20-15$ & 87,7 & 68,3 & 43 & 4,9 & 0,245 \\
10 & 15 & 87,7 & 60 & 28 & 3,3 & 0,220 \\
11 & 8 & 87,7 & 77,7 & 66 & 8 & 1,000 \\
12 & 10 & 87,7 & 83,8 & 88 & 13 & 1,300 \\
\hline
\end{tabular}

Tabla 2. Programa de secado suave.

\begin{tabular}{ccccccc}
\hline Etapa & $\% \mathrm{CH}$ & $\mathrm{TBS}\left({ }^{\circ} \mathrm{C}\right)$ & $\mathrm{TBH}\left({ }^{\circ} \mathrm{C}\right)$ & $\% \mathrm{HR}$ & $\mathrm{CHE}$ & $\mathrm{CS}$ \\
\hline 0 & - & 60 & 60 & 100 & - & - \\
1 & $120-70$ & 60 & 56,1 & 82 & 13,8 & 0,230 \\
2 & $70-60$ & 60 & 54,4 & 75 & 11,9 & 0,238 \\
3 & $60-50$ & 60 & 51,6 & 64 & 9,6 & 0,240 \\
4 & $50-40$ & 60 & 48,8 & 54 & 8,0 & 0,228 \\
5 & $40-35$ & 60 & 46,11 & 46 & 6,9 & 0,230 \\
6 & $35-30$ & 65,5 & 48,8 & 41 & 5,8 & 0,232 \\
7 & $30-25$ & 71,1 & 51,6 & 37 & 5,1 & 0,255 \\
8 & $25-20$ & 76,6 & 57,2 & 39 & 5,0 & 0,333 \\
9 & $20-15$ & 82,2 & 54,4 & 26 & 3,3 & 0,220 \\
10 & 15 & 82,2 & 72,2 & 65 & 8 & 1 \\
11 & 8 & 82,2 & 78,3 & 85 & 13 & 1,300 \\
\hline
\end{tabular}

Tabla 3. Diseño factorial 22 para el secado de Pinus pseudostrobus.

\begin{tabular}{ccc}
\hline Prueba & Programa & $\begin{array}{c}\text { Velocidad de aire } \\
(\mathrm{m} / \mathrm{s})\end{array}$ \\
\hline 1 & Suave & 3,35 \\
2 & Acelerado & 3,35 \\
3 & Acelerado & 2,05 \\
4 & Suave & 2,05 \\
5 & Suave & 2,05 \\
6 & Suave & 3,35 \\
7 & Acelerado & 3,35 \\
8 & Acelerado & 2,05 \\
\hline
\end{tabular}


Tabla 4. Porcentaje de valores permisibles de defectos para una muestra de $2,5 \times 20 \times 50 \mathrm{~cm}$.

\begin{tabular}{|c|c|c|c|c|c|c|}
\hline \multirow{2}{*}{\multicolumn{2}{|c|}{ Defecto }} & \multirow[t]{2}{*}{$\begin{array}{l}\text { Características a medir } \\
\text { respecto a: }\end{array}$} & \multicolumn{4}{|c|}{$\begin{array}{l}\text { Valor máximo permisible } \\
\text { en } \% \text { de su dimensión total }\end{array}$} \\
\hline & & & g1 & g2 & g3 & g4 \\
\hline \multicolumn{2}{|l|}{ Rajaduras } & al largo & 3,00 & 5,00 & 8,00 & $>800$ \\
\hline \multicolumn{2}{|l|}{ Grietas } & al largo & 10,0 & 20,00 & 30,00 & $>300$ \\
\hline \multicolumn{2}{|l|}{ Arqueadura } & al largo & 0 & 2,00 & 4,00 & $>400$ \\
\hline \multicolumn{2}{|l|}{ Encorvadura } & al largo & 0 & 1,00 & 1,50 & $>1,50$ \\
\hline \multicolumn{2}{|l|}{ Torcedura } & al largo & 0 & 1,00 & 1,50 & $>1,50$ \\
\hline \multicolumn{2}{|l|}{ Acanaladura } & al ancho & 0 & 0,50 & 1,00 & $>1,00$ \\
\hline \multicolumn{7}{|c|}{ Grados de calidad por factor de desclasificación, (Kauman y Mittak, 1996). } \\
\hline & Grado & \multicolumn{2}{|c|}{ Factor de desclasificación } & \multicolumn{3}{|c|}{ Intensidad de defecto } \\
\hline & 1 & 0,00 & \multicolumn{4}{|c|}{ Ausente } \\
\hline & 2 & 0,50 & \multicolumn{4}{|c|}{ Ligero } \\
\hline & 3 & 2,00 & \multicolumn{4}{|c|}{ De regular a fuerte } \\
\hline & 4 & 2,50 & \multicolumn{4}{|c|}{ Intenso } \\
\hline
\end{tabular}

Tabla 5. Clasificación de la calidad del secado (Kauman y Mittak, 1996).

\begin{tabular}{ccc}
\hline $\begin{array}{c}\text { MEDIA PONDERADA DE } \\
\text { DESCALIFICACIÓN (MPD) }\end{array}$ & $\begin{array}{c}\text { INDICE DE CALIDAD } \\
\text { DE SECADO (ICS) }\end{array}$ & $\begin{array}{c}\text { CONDICIONES DE } \\
\text { SECADO (CDS) }\end{array}$ \\
\hline 0,00 a 0,00 & Excelente & Adecuadas \\
0,01 a 0,50 & Muy buena \\
0,51 a 1,00 & Buena & \\
1,01 a 1,50 & Satisfactoria & Poco adecuadas \\
1,51 a 2,00 & Regular & \\
2,01 a 3,00 & Defectuosa \\
3,01 a 5,00 & Mala & Inadecuadas \\
Mayor de 5,00 & Muy mala & \\
\hline
\end{tabular}




\section{RESULTADOS Y DISCUSIÓN}

En la figura 2, se muestran las cinéticas de secado de cuatro experimentos. Se observa que el programa de secado suave entra a la fase de equilibrio en 30 horas, y el programa de secado acelerado lo hace en 20 horas; esta diferencia puede ser traducida en 10 horas de diferencia de consumo energético. Se observa también que la velocidad de aire tiene una ligera influencia en las cinéticas de secado en su fase capilar (eliminación del agua libre), pues el contenido de humedad disminuye en menor tiempo. Con un programa de secado acelerado la velocidad del aire muestra un efecto de aceleración; sin embargo, el contenido de humedad de equilibrio final no es afectado por una mayor velocidad de aire, observándose lo contrario para las condiciones de secado suaves. Los programas de secado acelerados son una opción para reducir los costos por consumo eléctrico ó uso de aceites térmicos y materiales de combustión usados en el calentamiento del aire de secado.

La figura 3 corresponde a la velocidad de secado. En esta figura se observa con claridad las etapas del secado de madera en función del Contenido de Humedad. En la primera etapa se observa un aumento de la rapidez de secado, debido al calentamiento del material; la segunda etapa corresponde a la pérdida de humedad por arriba del punto de saturación de la fibra; en esta etapa la migración de agua es principalmente afectada por fuerzas capilares; la tercera etapa consiste en acondicionar las especímenes de tal forma que se homogenice la humedad en el material, para disminuir esfuerzos que deformen el material (Bollman, 1984). En esta parte el equilibrio higroscópico establece la relación de rapidez de secado, pues las propiedades del material dominan sobre las condiciones externas.

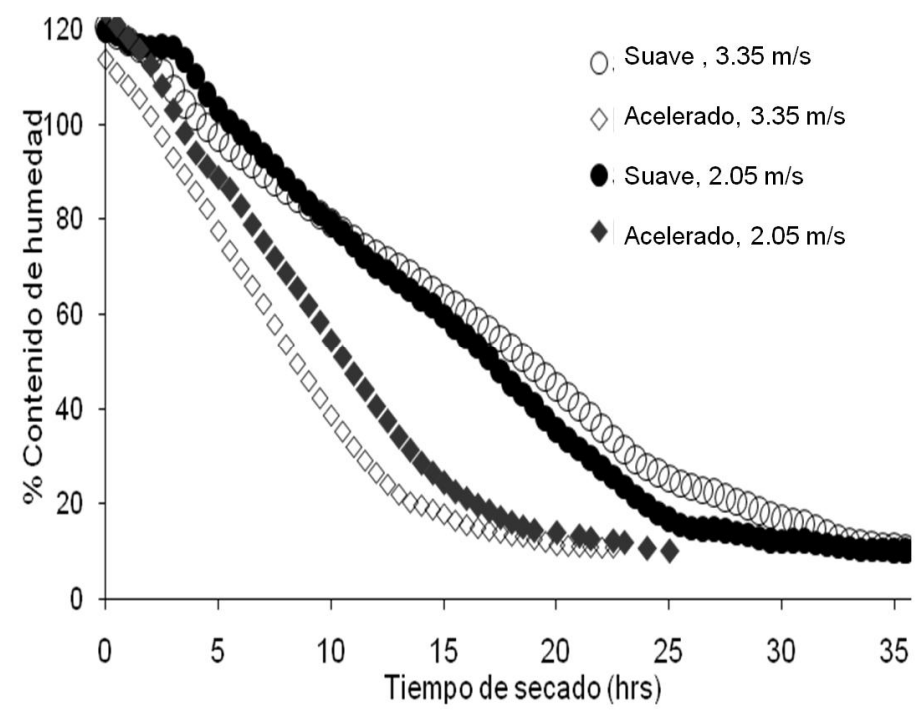

Figura 2. Cinética de dos programas de secado de pino pseudostrobus. 


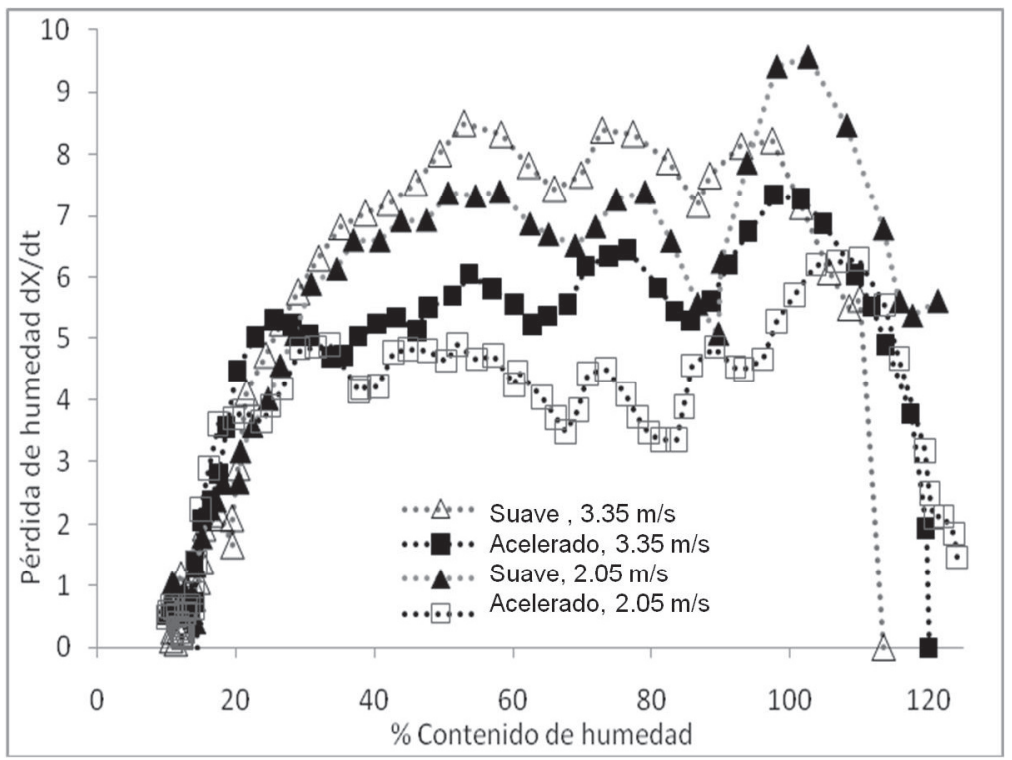

Figura 3. Perfil de la pérdida de humedad durante el secado de pino pseudostrobus.

En el tabla 6 se registraron los valores obtenidos de la media ponderada de defectos para clasificar la calidad de las especímenes y la calidad del secado, en las especímenes secas se observó una ligera acanaladura, lo que resulta en una condición de secado inadecuada (de acuerdo al método empleado) y en un índice de secado malo. Ahora bien, si no incluimos en el análisis este defecto por acanaladura, se pondera que la condición de secado es de inadecuado a poco adecuado, y el índice de secado de regular a malo, lo cual se debió también a que la madera usada fue de tercera calidad desde el inicio. En la práctica, este defecto por acanaladura es contrarestado colocando un sobrepeso en la pila de madera. Para comprobar ausencia o presencia de esfuerzos, se realizó una prueba de tenedor a las especímenes, que consistió en seccionar cada $0,5 \mathrm{~cm}$ una tabla de $2,5 \mathrm{~cm}$ de ancho obtenida de los extremos de las especímenes. Si las secciones no presentan deformación, el material está libre de esfuerzos. En nuestro trabajo, esta prueba se realizó por duplicado y se observó la ausencia de esfuerzos. Los resultados mostraron que las condiciones de secado intensas generan tablas de regular calidad y el secado es defectuoso, en cambio con condiciones de secado suaves la calidad del material y el secado se clasifican como malos.

En el análisis de varianza $(\alpha=0,05)$ se establecieron como factores el programa de secado $(P)$ y la velocidad de aire (VA). Se observó que el programa de secado combinado con la velocidad de aire influye en la calidad en la madera seca, sin embargo, en el tiempo de secado influye únicamente el programa de secado (Tabla 7). Por lo tanto la condición aceptable de acuerdo a nuestro estudio es aplicar un programa de secado acelerado con una velocidad de aire 3,35 $\mathrm{ms}^{-1}$ lo cual reduce más la presencia de defectos y el tiempo de secado. 
Tabla 6. Calidad del secado de acuerdo a la media ponderada de defecto.

\begin{tabular}{lccccc}
\hline PRUEBA & \multicolumn{3}{c}{ CON ACANALADURA } & SIN ACANALADURA \\
\hline & $\begin{array}{c}\text { Media ponderada } \\
\text { de defectos } \\
\text { (MPD) }\end{array}$ & $\begin{array}{c}\text { Condiciones } \\
\text { de secado } \\
\text { (CDS) }\end{array}$ & $\begin{array}{c}\text { Índice de calidad } \\
\text { de secado } \\
\text { (IDS) }\end{array}$ & $\begin{array}{c}\text { Condiciones } \\
\text { de secado } \\
\text { (CDS) }\end{array}$ & $\begin{array}{c}\text { Índice de calidad } \\
\text { de secado } \\
\text { (IDS) }\end{array}$ \\
\hline $\begin{array}{l}\text { Suave a } 3,35 \text { ms-1 } \\
\text { Acelerado y } 3,35 \text { ms-1 }\end{array}$ & 3,725 & Inadecuada & Mala & Inadecuada & Defectuosa \\
Acelerado y 2,05 ms-1 & 3,500 & Inadecuada & Mala & Poco adecuada & Regular \\
Suave y 2,05 ms-1 & 4,625 & Inadecuada & Mala & Inadecuada & Defectuosa \\
Suave y 2,05 ms-1 & 4,625 & Inadecuada & Mala & Inadecuada & Defectuosa \\
Suave y 3,35 ms-1 & 6,750 & Inadecuada & Muy mala & Inadecuada & Mala \\
Acelerado y 3,35 ms-1 & 3,750 & Inadecuada & Mala & Poco adecuada & Regular \\
Acelerado y 2,05 ms-1 & 4,625 & Inadecuada & Mala & Inadecuada & Defectuosa \\
\hline
\end{tabular}

Tabla 7. Análisis de Varianza obtenido del secado de pino chalamite.

\begin{tabular}{|c|c|c|c|c|c|c|}
\hline \multicolumn{7}{|c|}{ Calidad en madera seca } \\
\hline Origen & DF & $\begin{array}{c}\text { Suma de } \\
\text { cuadrados }\end{array}$ & $\begin{array}{l}\text { Media de } \\
\text { cuadrados }\end{array}$ & $\begin{array}{r}\text { Rotación } \\
\text { F }\end{array}$ & $\begin{array}{r}\text { Nivel de } \\
\text { Probabilidad }\end{array}$ & $\begin{array}{r}\text { Poder } \\
(\text { alfa }=0,05)\end{array}$ \\
\hline$P$ & 1 & 5,12 & 5,12 & 22,40 & $0,009086^{*}$ & 0,859140 \\
\hline VA & 1 & 1,015313 & 1,015313 & 4,44 & 0,122780 & 0,290147 \\
\hline P/VA & 1 & 2,152812 & 2,152812 & 9,42 & $0,037336^{*}$ & 0,522851 \\
\hline$S$ & 4 & 0,914375 & 0,2285938 & & & \\
\hline Ajuste total & 7 & 9,2025 & & & & \\
\hline Total & 8 & & & & & \\
\hline \multicolumn{7}{|c|}{ Tiempo de secado } \\
\hline Origen & DF & $\begin{array}{c}\text { Suma de } \\
\text { cuadrados }\end{array}$ & $\begin{array}{l}\text { Media de } \\
\text { cuadrados }\end{array}$ & $\begin{array}{r}\text { Rotación } \\
\text { F }\end{array}$ & $\begin{array}{r}\text { Nivel de } \\
\text { Probabilidad }\end{array}$ & $\begin{array}{r}\text { Poder } \\
(\text { alfa }=0,05)\end{array}$ \\
\hline$P$ & 1 & 479220,5 & 479220,5 & 6,91 & 0,058299 & 0,412761 \\
\hline VA & 1 & 51842 & 51842 & 0,75 & 0,436115 & 0,090254 \\
\hline P/VA & 1 & 32258 & 3225,8 & 0,46 & 0,532759 & 0,074954 \\
\hline$S$ & 4 & 277519 & 69379,75 & & & \\
\hline Ajuste total & 7 & 840839,5 & & & & \\
\hline Total & 8 & & & & & \\
\hline
\end{tabular}

* termino significativo de alfa $=0,05$ 
Los resultados obtenidos se compararon con estudios realizados para otras especies de pino. Milota et al (2000) estudiaron el efecto de las temperaturas altas en la formación de alabeos en pino amarillo del sur (SYP), los autores observaron que el secado a altas temperaturas reduce significativamente la formación de alabeos y por ende el tiempo de secado. Fuentes, (1991); Guerrero, (1980); y Pérez et al., (2007), mencionan que el defecto común en madera es la encorvadura, el cual desclasifica la madera y la puede colocar en un cuarto ó quinto nivel de calidad, además de que otro factor que influye en esta desclasificación es el reducido número de especímenes evaluadas.

\section{CONCLUSIÓN}

Los programas de secado aplicados en este estudio, permiten tener mayor información acerca de efecto de los variables de secado. Los resultados son aceptables en el sentido que se alcanza un contenido de humedad de equilibrio acorde a las especificaciones para su comercialización. El diseño factorial $2^{2}$ mostró que la condición de secado mas apropiada para Chalamite (Pinus pseudostrobus) fue el programa de secado acelerado con velocidad de aire de $3,35 \mathrm{~ms}^{-1}$, pues reduce el tiempo de secado y la presencia de defectos. Una clara diferencia en los tiempos de secado fue observado entre los programas de secado intensos $y$ suaves, lo cual esta directamente relacionado con el nivel de temperatura; esta diferencia de tiempo de secado es importante desde un punto de vista económico, pues el consumo energético de una estufa de secado tradicional es siempre parámetro a minimizar. La calidad de la madera especímenes secas indicó una condición de secado inadecuada y una calidad defectuosa en la madera, lo cual a nivel industrial o laboratorio se podrá contrarrestar en próximos estudios con el uso de madera de primera calidad y aplicar el sobrepeso colocado en las pilas de madera.

\section{AGRADECIMIENTOS}

Se agradece el apoyo financiero al Instituto Politécnico Nacional (proyecto CGPI2003-34), al SIBEJ-CONACYT (proyecto 19990505013), a la Unión Forestal "IXETO" por el apoyo recibido y a OSMOSE Mexicana por la asesoría técnica.

\section{REFERENCIAS}

Álvarez, H. y J. I. Fernández. 1992. Fundamentos teóricos del secado de la madera. MAP, Madrid p. 32-35.

Aytekin, A., G. Gunduz, B. Kaygin, S. Korkut y S.M. Onat. 2009. Drying Schedules calculations of Caminayan, Blank Pine (Pinus nigra Arn. subsp. pallasiana var. pallasiana) by computer programming. African Journal of Biotechnology 8(8):1703-1712.

Blakemore, P.A., Langrish, T.A.G. 2008. Effect of collapse of fitted diffusion cofficients for Victorian ash eucalypts. Journal Wood Science and Technology. 42:535-549.

Bollman, L. 1984. Manual para secado de madera. Luwing Bollman K.G. Manshinienfabrik. República Federal Alemana p 440.

Brooke, A.S. and T. A. G. Langrish. 1997. The simulation of stresses and strains in the drying of Pinus radiata sapwood: the effects of board geometry. Computers and Chemical Engineering. 21 (11): 1271-1281.

Bustos, C., R.E. Hernandez y Y. Fortin. 2009. Effect of kiln-drying on the hard- 
ness and machining properties of tamarack wood for flooring. Forest Products Journal. 59(1-2):71-76.

Carlsson, P. y M. Tinnsten. 2002. Optimization of drying schedules for a mixture of boards with distribution of sapwood and heartwood. Drying Technology. 20(2):403-418.

Fuentes-Salinas, M. 1991. Secado de madera de Pinus radiata $S$. Don impregnada con sales hidrosolubles CCA. Tesis de maestría Universidad Austral de Chile Valdivia, Chile, p 162.

Guerrero, M. G. 1980. Comportamiento de tablas de Eucaliptos globulus labill, crecido en Culcura, sometidas a un programa de secado australiano. Convenio UACH/Sociedad Agrícola y Forestal Colcura S. A. Facultad de Ingeniería Forestal. Informe de Convenio No. 25.

Instituto Nacional de Normalización (INN). 1993. Madera, procedimientos y criterios de evaluación para clasificación. Norma Chilena 993 EOf 72 Santiago Chile.

Jankowsky, I. P. 1995. Equipamentos e processos para a secagem de madeiras. Procedem of. Seminário Internacional de utilização da madeira de Eucalipto para serraria. IPEF piracicaba. p 109-118.

Kauman, W.G., y G. Mittak. 1966. The effect of thermal degradation on shrinkage and collapse of wood from 3 Australian species, Forests Product Journal, 11(9):445-452.

Kollmann F. F. P y W. A. Cotê. 1968. Principles of Wood Science and Technology. V. I. Solids wood. Springer Verlag, Berlin p. 214.
Langrish, T.A.G., A.S Brooke, C.L Davis, H.E Mush, y G.W. Barton. 1997. An improved drying schedule for Australian iron bark timber: Optimization and experimental validation. Drying Technology. 15(1):47-70.

Milota M.R., 2000. Warp and shrinkage of hem-fir stud lumber dried at conventional and high temperatures. Forest Products Journal 50(11/12):79-84.

Pérez P., R.A. Ananías y G. Hernández. 2007. Estudio experimental del secado de renovales de canelo Drymis winteri. Maderas, Ciencia y tecnología 9(1):5970.

Rodríguez R.J, L.L. Méndez, A.C. Martínez y N.F. Diego. 2001. A closed loop tunnel for drying-kinetics research. InterAmerican Drying Conference, Boca del Río Veracruz, México p. 499-504.

Rosen H. N, 1983. Recent advances in the theory of drying lumber. Proceedings of the IUFRO. Division V Conference, Southern Illinois University, USDA, Madison. p 32-64.

Sandoval-Torres, S. 2009. Modelación matemática del secado convencional de madera. Madera y Bosques 15(1):75-89.

Sandoval-Torres, S., J. Rodríguez-Ramírez, L.L. Méndez-Lagunas y M. FuentesSalinas. 2004. Flujo de aire sobre el proceso de secado de madera de encino (Quercus Candicans Nee). Información Tecnológica 15 (1):11-16.

Simpson, W.T. 1994. Grouping tropical wood species for kiln drying using mathematical models. Drying Technology 12(8):1877-1896.

Simpson, W.T., 1996, Method to estimate dry-kiln schedules and 
species groupings: Tropical and temperate hardwoods. USA Forest Service, FPRL Madison Res. Pap. FPL-RP-549-557 pp.

http://www.fpl.fs.fed.us/documnts/fplrp/fplrp5 48.pdf

Solís Rodríguez, L.E., M.A. Cerón Cardeña e I. González Ahumada. 2003. Diseño y operación de una estufa solar para secar madera. Revista Ingeniería: 35-48.
USAID. 2007. Empresas Forestales Comunitarias en Países Forestales Tropicales. Situación Actual y en Potencia. Octubre, p 30-35.

Wiberg, P., S.M.B. Sehlstedt, y T.J Morén. 2000. Heat and mass transfer during sapwood drying above the fiber saturation point. Drying Technology: An International Journal, 1532-2300, 18(8):1647-1664. 